\title{
An Experimental Assessment \\ of Composite Mechanical Properties \\ in a Filament-Wound Pressure Vessel
}

\author{
Egor V. Moskvichev* \\ SDTB "Nauka” ICT SB RAS
}

P.O. box 25515, Krasnoyarsk, 660049, Russia

Received 16.11.2015, received in revised form 17.01.2016, accepted 12.02.2016

The results of the assessment of composite mechanical properties in a filament-wound pressure vessel are presented. The cutting diagram, the method of sample preparation and testing procedure are shown. The comparison of the elastic modulus determined on three-point bending tests and tensile tests was carried out.

Keywords: spacecraft, filament-wound pressure vessel, composite material, testing, mechanical properties.

Citation: Moskvichev E.V. An experimental assessment of composite mechanical properties in a filament-wound pressure vessel, J. Sib. Fed. Univ. Eng. technol., 2016, 9(2), 246-253, DOI: 10.17516/1999-494X-2016-9-2-246-253.

\section{Экспериментальная оценка характеристик \\ механических свойств материала \\ композитной оболочки металлокомпозитного бака \\ высокого давления}

Е.В. Москвичев

СКТБ «Наука» ИВТ СО РАН

Россия, 660049, Красноярск, а/я 25515

Приведены результаты испытаний по оценке характеристик механических свойств материала композитной оболочки металлокомпозитного бака высокого давления. Обоснована схема вырезки и способ подготовки образиов, изложена методика их испытаний. Приведено сравнение значений продольного модуля упругости, определенных методом испытаний на трехточечный изгиб прямоугольных образиов и методом испытаний на растяжение образиов из композитной ленты.

(C) Siberian Federal University. All rights reserved

* Corresponding author E-mail address: jugr@icm.krasn.ru 
Ключевые слова: космические аппараты, металлокомпозитный бак высокого давления, композиционные материаль, испытания, механические свойства.

\section{Введение}

К современным космическим аппаратам тяжелого класса предъявляются высокие требования по весовой эффективности и сроку активного существования. Одним из показателей, определяющих соответствие данным требованиям, является запас топлива для электрореактивных двигателей, выводящих космический аппарат на геостационарную орбиту и корректирующих его положение в процессе эксплуатации. В связи с этим применение композитных баллонов давления вызывает особый интерес у разработчиков космической техники, поскольку композитные баллоны позволяют при меньшей собственной массе вместить большую массу топлива. Это достигается путем хранения топлива при высоких давлениях, что обусловливает необходимость тщательного рассмотрения задач прочности и надежности композитных баллонов.

В статье представлены результаты исследований материала композитной оболочки металлокомпозитного бака высокого давления (МКБВД), разработанного предприятием АО «Информационные спутниковые системы» имени академика М.Ф. Решетнёва. Данный МКБВД при собственной массе 34 кг вмещает до 350 кг ксенона при рабочем давлении 8 МПа, что позволяет обеспечивать топливом двигатели системы коррекции спутников тяжёлого класса в течение срока не менее 15 лет.

МКБВД представляет собой осесимметричный сосуд с внутренним объемом 350 литров, состоящий из титанового лейнера, обеспечивающего герметичность, и силовой композитной оболочки, обеспечивающей прочность и жесткость (рис. 1). Силовая композитная оболочка изготавливается методом многослойной непрерывной намотки на лейнер пакетов угольных волокон IMS60, пропитанных связующим ЭД-И.

Учитывая высокую ответственность конструкции, задачи обеспечения прочности и надежности МКБВД являются приоритетными в процессе его разработки и изготовления. Для решения этих задач проводится комплексный анализ прочности и герметичности, включаю-

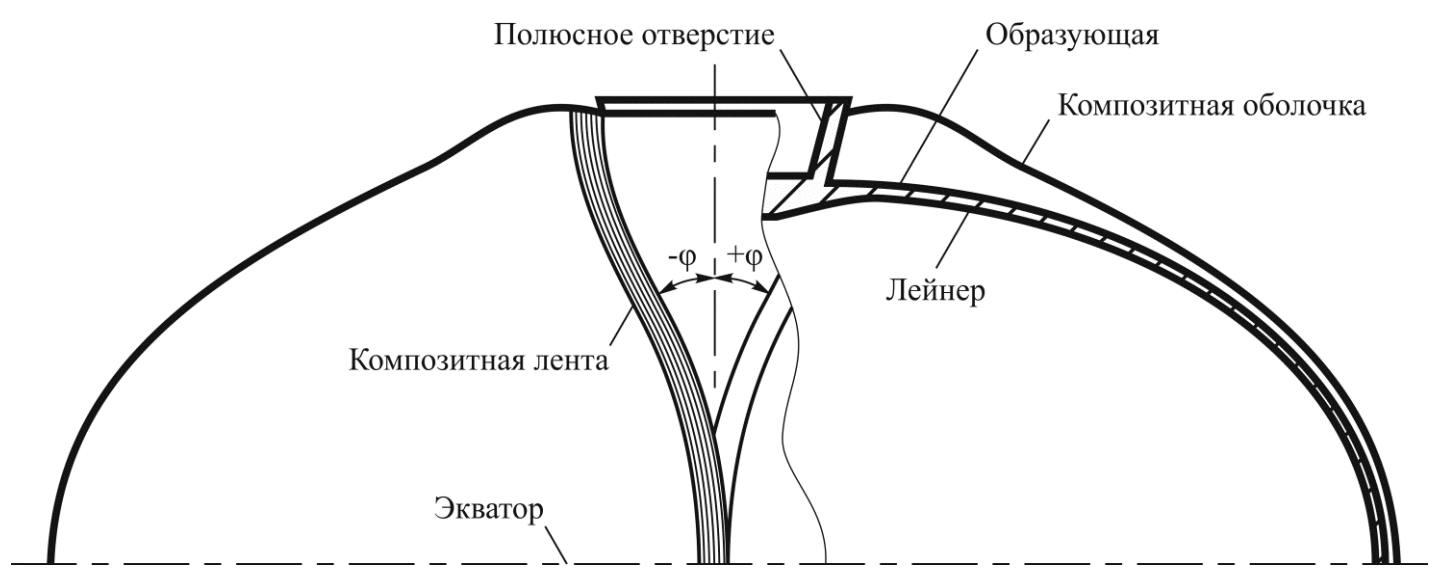

Рис. 1. Металлокомпозитный бак высокого давления

$$
-247-
$$


щий экспериментальные и расчетные обоснования $[1,2]$. При этом особое внимание уделяется характеристикам механических свойств материалов, применяемых в МКБВД, поскольку в условиях длительной эксплуатации необходимо обеспечить не менее чем двукратный запас прочности. Это выдвигает высокие требования к механическим свойствам материала силовой композитной оболочки.

Прочностные и деформационные свойства композитной оболочки в значительной степени определяются не только механическими свойствами угольных волокон и эпоксидной матрицы, входящими в состав материала, но и технологическими параметрами намотки. Таким образом, для оценки фактической жесткости и прочности композитной оболочки необходимо проведение экспериментальных исследований по определению характеристик механических свойств композитного материала, сформированного непосредственно в процессе изготовления МКБВД, что и являлось целью данной работы.

\section{Структура материала}

Материал композитной оболочки имеет слоистую структуру, состоящую из композитных лент (пакетов волокон), уложенных друг на друга. Каждый слой формируется двумя лентами, симметрично повернутыми под углом $\pm \varphi$ относительно образующей оболочки (рис. 2a). При этом угол укладки $\varphi$ не является постоянным, а изменяется вдоль образующей согласно геодезической траектории намотки и определяется формулой Клеро. В процессе укладки по мере приближения к полюсному отверстию и уменьшения радиуса композитная лента постепенно перекрывает нижележащие слои, образуя композитную оболочку переменной толщины. Наименьшая толщина композитной оболочки наблюдается на экваторе, а наибольшая в зоне полюсного отверстия.

Согласно данной схеме намотки материал композитной оболочки можно рассматривать как ортотропный с девятью механическими характеристиками, которые зависят от угла $\varphi$ : тремя модулями упругости $\left(E_{x}, E_{y}, E_{z}\right)$, тремя модулями сдвига $\left(G_{12}, G_{13}, G_{23}\right)$ и тремя коэффициентами Пауссона $\left(\mu_{12}, \mu_{13}, \mu_{23}\right)$. Экспериментальное определение девяти механических характеристик при различных углах укладки на практике не представляется возможным. Однако соотношения структурной механики композитных материалов позволяют произвести расчет

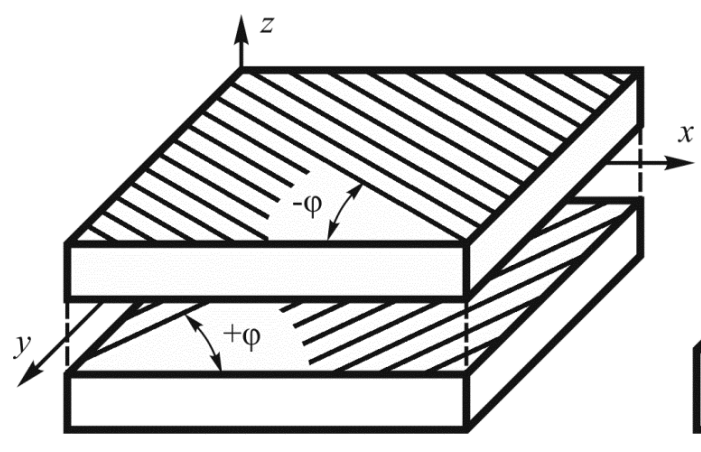

a)

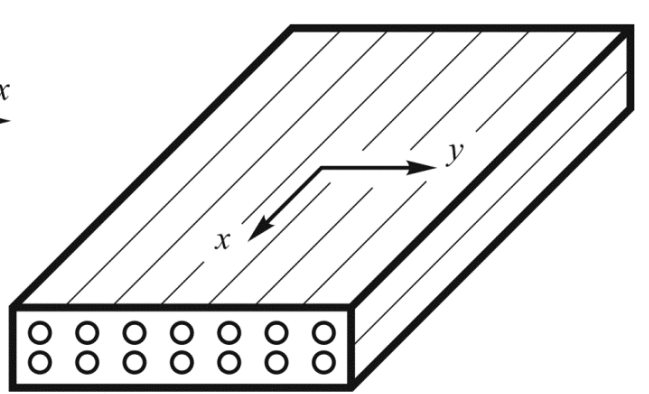

б)

Рис. 2. Структура материала композитной оболочки со схемой армирования $[-\varphi /+\varphi]_{\mathrm{n}}$

$$
-248-
$$


этих характеристик на основе механических свойств композитной ленты, формирующей композитную оболочку в процессе намотки [3].

Композитная лента состоит из параллельных однонаправленных угольных волокон, равномерно распределенных по сечению и пропитанных связующей эпоксидной смолой (рис. 2б). Материал такой структуры характеризуется как трансверсально-изотропный (или поперечно изотропный), свойства которого существенно отличаются в двух взаимно перпендикулярных направлениях и определяются пятью механическими характеристиками: $E_{x}, E_{y}, \mu_{12}, \mu_{23}$ и $G_{12}$. В случае плосконапряженного состояния, когда толщина композитной ленты намного меньше ее ширины, количество механических характеристик сокращается до четырех: $E_{x}, E_{y}, \mu_{12}$ и $G_{12}$, экспериментальное определение которых возможно посредством проведения стандартных испытаний. В работе определялись значения модулей упругости $E_{x}, E_{y}$ и предела прочности $\sigma_{u}$ однонаправленного композита.

Для экспериментальной оценки характеристик механических свойств композиционной ленты наиболее предпочтительными являются испытания однонаправленно армированных образцов, изготовленных по той же технологии, что и композитная оболочка. Такие испытания позволяют наиболее достоверно оценить полный набор необходимых характеристик. Однако когда отсутствует возможность изготовления однонаправленных образцов, они вырезаются непосредственно из конструкции. В таком случае зона вырезки образцов является особенно критичной, поскольку исследуемые характеристики зависят от угла укладки волокон $\varphi$.

Характер изменения механических свойств в различных зонах композитной оболочки можно оценить по результатам аналитического расчета эквивалентной матрицы жесткости многослойного композита в зависимости от угла укладки $\varphi$ [4]. На основе характеристик механических свойств композитной ленты, представленных в работе [5] для аналогичного композиционного материала, были построены функции изменения продольного $E_{x}$ и поперечного $E_{y}$ модуля упругости (рис. 3).

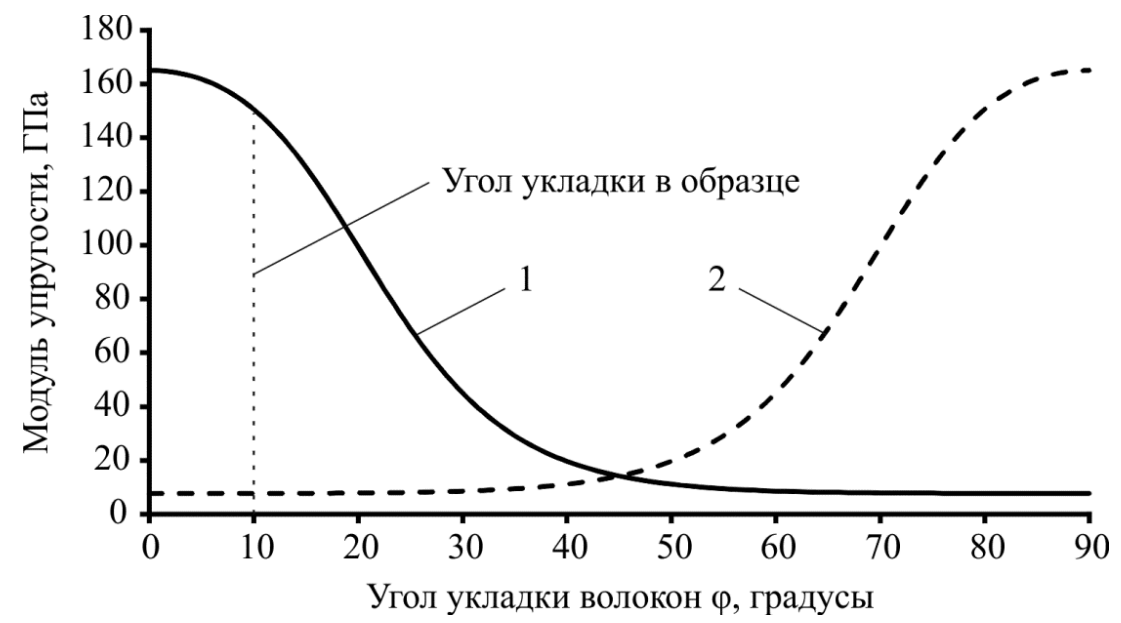

Рис. 3. Характер изменения продольного $E_{x}(1)$ и поперечного $E_{y}(2)$ модулей упругости в зависимости от угла укладки $\varphi$ 
При небольших углах укладки (до 10 градусов) значения продольного и поперечного модулей упругости изменяются слабо. Таким образом, в исследуемой композитной оболочке МКБВД наиболее подходящей областью для вырезки образцов является зона экватора, в которой угол укладки является минимальным и составляет 10 градусов, следовательно, механические характеристики, определяемые на таких образцах, наиболее приближены к характеристикам однонаправленного композита.

\section{Подготовка образцов}

Для оценки продольного $E_{x}$ и поперечного $E_{y}$ модулей упругости было изготовлено соответственно десять вертикальных и десять горизонтальных прямоугольных образцов для испытаний на трехточечный изгиб. Образцы вырезались симметрично относительно линии экватора согласно разметке (рис. 4). Размеры образцов и схема нагружения представлены на рис. 5. Измерения толщины образцов $h$ проводились в 12 точках вдоль образца. Для вертикальных образцов средняя толщина составила 3,12 мм, для горизонтальных - 3,06 мм. Радиус кривизны $R$ для горизонтальных образцов соответствовал радиусу экватора и равнялся 506 мм. Для вертикальных образцов радиус кривизны определялся формой образующей и равнялся 236 мм.

В дополнение к испытаниям прямоугольных образцов были проведены испытания на растяжение двадцати образцов из композитной ленты для оценки продольного модуля упругости $E_{x l}$ и предела прочности $\sigma_{u}$. Для получения образцов фрагменты ленты отслаивались с внешней поверхности оболочки с помощью режущего инструмента. На концы ленты приклеивались пластинки из стекловолокна для жесткого закрепления образцов в захватах испытательной машины и исключения проскальзывания (рис. 6). Толщина композитных лент $h$ изменялась от 0,18 до 0,33 мм, ширина $b$ от 4 до 16 мм. Рабочая часть образцов $l_{0}$ составляла от 30 мм до 60 мм в зависимости от полной длины образца.

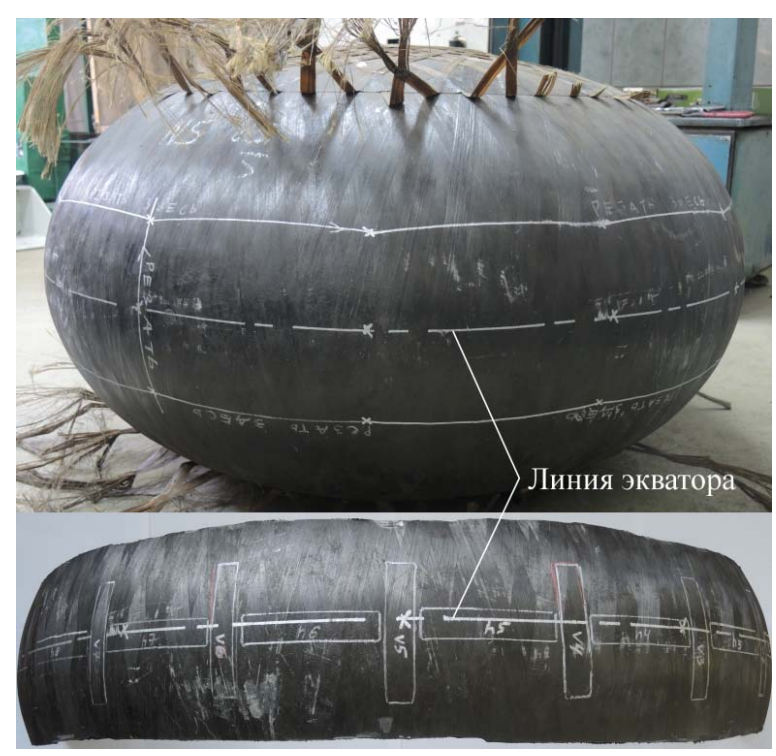

Рис. 4. Разметка зон для вырезки образцов из МКБВД 

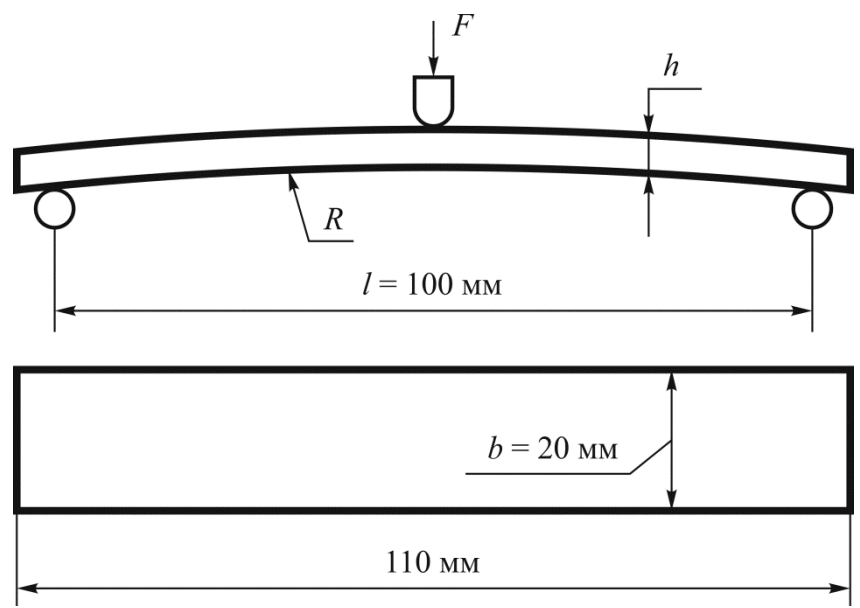

Рис. 5. Размеры и схема нагружения прямоугольных образцов

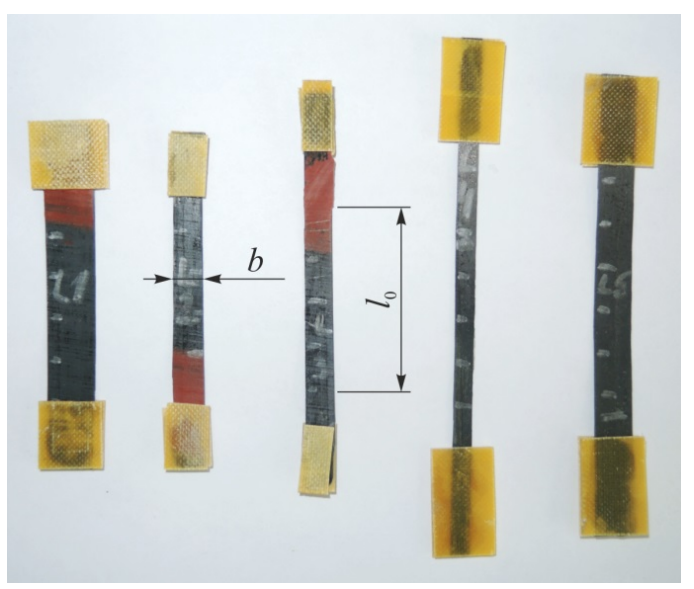

Рис. 6. Образцы композитной ленты

\section{Испытания и результаты}

Испытания проводились на электромеханической испытательной машине Zwick/Roell Z100 с использованием приспособления для трехточечного изгиба. При испытании на растяжение применялись клиновидные захваты и внешний контактный экстензометр. В процессе испытаний фиксировались диаграммы «нагрузка-прогиб» при изгибе (рис. 7а) и «нагрузкаудлинение» при растяжении (рис. 7б).

Обработка результатов испытаний на изгиб проводилась согласно ГОСТ 25.604-82 с учетом поправки на кривизну образцов, представленной в работе [6]. Продольный модуль упругости $E_{x}$ для вертикальных образцов и поперечный модуль упругости $E_{y}$ для горизонтальных образцов определялись с учетом их геометрических размеров по формуле

$$
E_{x(y)}=\frac{\Delta F \cdot l^{3}}{4 \cdot b \cdot h^{3} \cdot \Delta w}
$$


где $\Delta F$ - приращение нагрузки, $l$ - расстояние между опорами, $b$ - ширина образца, $h$ - толщина образца, $\Delta w$ - приращение прогиба в середине образца, соответствующее приращению нагрузки $\Delta F$.

Продольный модуль упругости и предел прочности композитной ленты определялись по ГОСТ 25.601-80:

$$
E_{x l}=\frac{\Delta F \cdot l_{0}}{b \cdot h \cdot \Delta l} ; \sigma_{\mathrm{u}}=\frac{F_{\max }}{b \cdot h},
$$

где $\Delta F$ - приращение нагрузки, $l_{0}$ - длина рабочей части, $b$ - ширина образца, $h$ - толщина образца, $\Delta l$ - приращение длины, соответствующее приращению нагрузки $\Delta F, F_{\max }$ - максимальная нагрузка, предшествующая разрушению.

По результатам эксперимента были определены средние значения и коэффициенты вариации исследуемых величин (табл. 1). Приведенные данные показывают, что продольный модуль упругости композитной ленты $E_{x l}$ близок по среднему значению к продольному модулю упругости $E_{x}$, определенному на прямоугольных образцах, но при этом имеет большую вариацию. Это объясняется тем, что жесткость тонких композитных лент в большей степени определяется объемным содержанием волокна, чем площадью поперечного сечения, измеренного в процессе испытаний. Таким образом, испытания композитных лент могут давать заниженную оценку продольного модуля упругости, что и наблюдается в эксперименте.

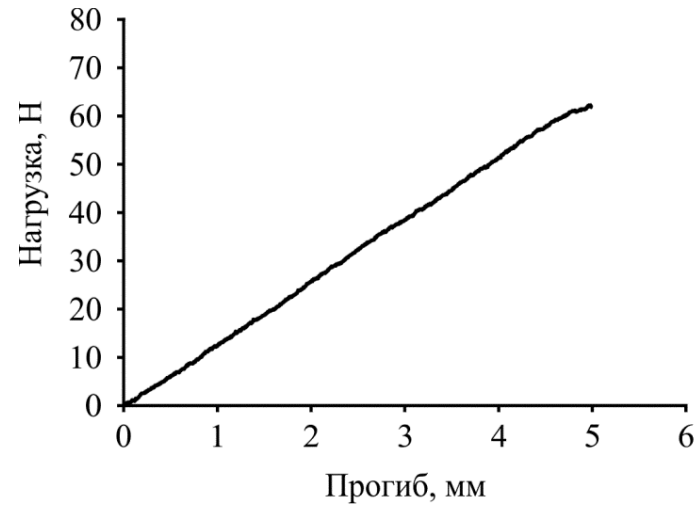

a)

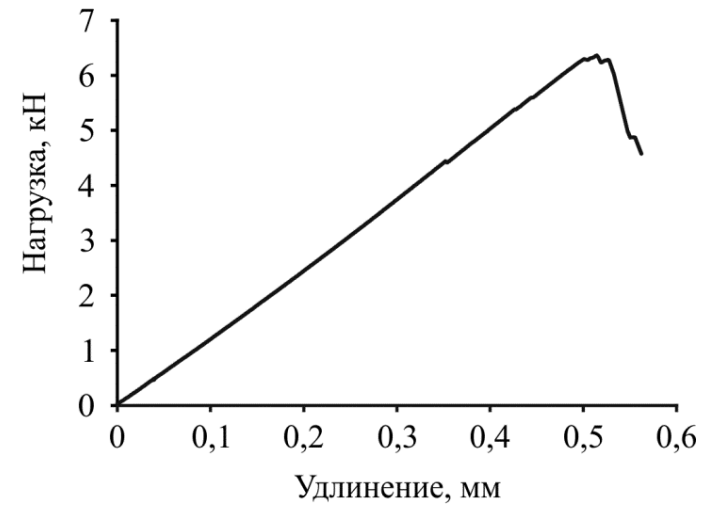

б)

Рис. 7. Диаграммы нагружения образцов при изгибе (а) и при растяжении (б)

Таблица 1. Экспериментальные значения модулей упругости и предела прочности

\begin{tabular}{|c|c|c|c|c|}
\hline Характеристика & $E_{x}$ & $E_{y}$ & $E_{x l}$ & $\sigma_{u}$ \\
\hline Среднее, МПа & 118138 & 5881 & 115702 & 2334 \\
\hline Вариация & 0,06 & 0,069 & 0,229 & 0,21 \\
\hline
\end{tabular}




\section{Заключение}

Полученные значения характеристик механических свойств отличаются от значений аналогичных по составу композитных материалов, представленных в [5]. Это не является противоречием, поскольку, как отмечалось выше, механические свойства композитной оболочки как элемента конкретной рассмотренной конструкции определяются не только механическими свойствами составляющих компонентов (волокон и матрицы), а в значительной мере такими технологическими параметрами изготовления, как траектория намотки, схема армирования, процентное содержание волокон, которые являются индивидуальными для МКБВД.

Проведенные эксперименты дают возможность оценивать характеристики механических свойств материала композитной оболочки на образцах, полученных непосредственно из МКБВД. Такие оценки позволяют проводить сравнительный анализ качества изготовления композитной оболочки в целях оптимизации технологических параметров и обеспечения стабильности прочностных свойств. Значения характеристик механических свойств также могут быть использованы при моделировании напряженно-деформированного состояния МКБВД и расчетах на прочность. Однако для повышения достоверности результатов необходимо проведение комплексных экспериментальных исследований по определению расширенного набора свойств на различных образцах при различных условиях нагружения.

Статья подготовлена по результатам исследований, проведенных при финансовой поддержкке Минобрнауки России в ходе реализации проекта по Соглашению № 14.607.21.0038 в рамках ФЦП «Исследования и разработки по приоритетным направлениям развития научно-технологического комплекса России на 2014-2020 годы» RFMEFI60714X0038.

\section{Список литературы}

[1] Lepikhin A.M., Burov A.E., Moskvichev V.V. Possibilities of the design estimates of the reliability of a high-pressure metal-composite tank. Journal of machinery manufacture and reliability, 2015, 44(4), 344-349.

[2] Лепихин А.М., Москвичев В.В., Черняев А.П., Похабов Ю.П., Халиманович В.И. Экспериментальная оценка прочности и герметичности металлокомпозитных сосудов высокого давления. Деформация и разрушение материалов, 2015, 6, 30-36 [Lepikhin A.M., Moskvichev V.V., Chernyaev A.P., Pohabov Y.P., Khalimanovich V.I. Experimental evaluation of strength and tightness of the metal-composite high-pressure vessels. Deformation and fracture of materials, 2015, 6, 30-36 (in Russian)].

[3] Vasiliev V.V. Composite Pressure Vessels: Analysis, Design, and Manufacturing. Blacksburg, 2009. 704 p.

[4] Barbero E.J. Finite elementan alysis of composite materials. CRC Press, Taylor \& Francis Group, 208. 331 p.

[5] Yoshimura A., Okabe T., Yamada M., Ogasawara T., Tanabe Y. Damage simulation of CFRP laminates under high velocity projectile impact. ICCM-18, S. Korea, 2011.

[6] Полилов А.Н. Экспериментальная механика композитов. М.: Издательство МГТУ им. Н.Э. Баумана, 2015. 375 с. [Polilov A.N. Experimental mechanics of composites. Moscow, The Bauman University Publishing House, 2015375 p. (in Russian)]

$$
-253-
$$

